\title{
EFECTO DE LA GRAVEDAD DEL TRASTORNO RESPIRATORIO DEL SUEÑO SOBRE EL DESEMPEÑO COGNITIVO EN UNA COHORTE DE NIÑOS EN EDAD ESCOLAR
}

\author{
EFFECT OF SLEEP-DISORDERED BREATHING SEVERITY ON COGNITIVE PERFORMANCE \\ MEASURES IN A LARGE COMMUNITY COHORT OF YOUNG SCHOOL-AGED CHILDREN
}

Hunter SJ, Gozal D, Smith DL, Philby MF, Kaylegian J, Kheirandish-Gozal L.

Am J Respir Crit Care Med 2016;194(6):739-747

Tal como ya se ha mencionado, los trastornos respiratorios del sueño (TRS)se asocian con una alto riesgo de desarrollar alteraciones cognitivas en los niños. Sin embargo no está claro si la asociación es dependiente de la severidad del TRS y/o si existe un punto de corte de la severidad del TRS para que aparezcan estos problemas.

Para lograr demostrar que si existe relación entre TRS y función cognitiva los autores proponen evaluar en forma prospectiva niños desde la edad pre-escolar, estudiando 1010 niños roncadores y no-roncadores desde los 5-7 años. Mediante cuestionario se determinan sus hábitos de sueño, se les realiza a todos polisomnograma y evaluación neurocognitiva (funciones intelectual,atención, memoria, lenguaje).

Según su polisomnograma los niños se clasificaron en 4 grupos:

1. No roncadores, con índice de apnea-hipopnea (AHÍ) menor de 1/hora durante el tiempo total de sueño (TST)

2. Roncadores habituales con AHÍ menor de 1/hora en TST

3. Roncadores habituales con AHÍ mayor de 1 pero menor de 5/hora de TST

4. Roncadores habituales con AHÍ mayor de 5/hora

El grupo 4 mostró un IMC significativamente mayor que los otros, mayor número de apneas obstructivas, menor nadir de Sp02, mayor índice de microdespertares. Los niños con mayores índices de apnea-hipopnea presentaron menor rendimiento en los tests de habilidad verbal y no-verbal, también en habilidad conceptual global y menor capacidad de resolver problemas.

Este sería el primer estudio que confirma el impacto de los TRS en el desarrollo neurocognitivo. Con estos hallazgos debieran proponerse políticas sanitarias para pesquisar precozmente los niños en riesgo. 